\title{
Sentido de coherencia y consumo de alcohol en jóvenes universitarios
}

\author{
Sense of coherence and alcohol consumption \\ in university students
}

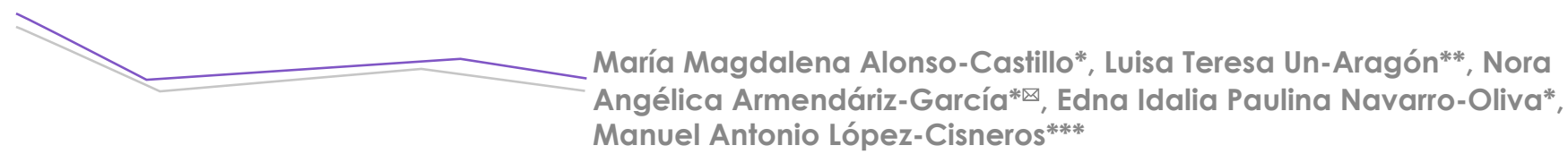

Alonso-Castillo, M. M., Un-Aragón, L. T., Armendáriz-García, N. A., Navarro-Oliva, E. I. P. \& López-Cisneros, M. A. (2018). Sentido de coherencia y consumo de alcohol en jóvenes universitarios. Investigación y Ciencia de la Universidad Autónoma de Aguascalientes, 26(75), 66-72.

\section{RESUMEN}

El sentido de coherencia es uno de los factores de protección que está teniendo una especial importancia en la prevención del uso de drogas. Resulta relevante conocer la relación entre el sentido de coherencia y el consumo de alcohol en los jóvenes universitarios. El diseño fue descriptivo correlacional con muestreo probabilístico estratificado, la muestra final fue de 227 jóvenes inscritos en una universidad privada. Las mujeres reportaron mayor consumo de bajo riesgo y de riesgo $(30.3 \%$ y $33.3 \%$, respectivamente) en comparación con los hombres, los cuales reportaron mayor consumo perjudicial (59.1\%). Se identificó una correlación negativa y significativa entre el sentido de coherencia y el consumo de al-

Palabras clave: coherencia; bebidas alcohólicas; estudiantes; jóvenes; prevención; protección.

Keywords: coherence; alcoholic beverages; students; young; prevention; protection.

\section{Recibido: 16 de mayo de 2018, aceptado: 6 de agosto de 2018}

* Subdirección de Posgrado e Investigación, Facultad de Enfermería, Universidad Autónoma de Nuevo León. Av. Gonzalitos \# 1500 Nte., Mitras Centro, C. P. 64460, Monterrey, Nuevo León, México. Correo electrónico: magdalena_alonso@hotmail.com; nordariz@hotmail.com; eipno@yahoo.com.mx

** Enfermería, Clínica Carranza. Venustiano Carranza \#366, Chetumal, C. P. 77035, Quintana Roo, México. Correo electrónico: luisateresa.un@ gmail.com

*** Facultad de Ciencias de la Salud, Universidad Autónoma del Carmen. Av. Concordia, Col. Benito Juárez, C. P. 24167, C. Carmen, Campeche, México. Correo electrónico: mlopez@pampano.unacar.mx

$\bowtie$ Autor para correspondencia cohol medido por el AUDIT $\left(r_{s}=-.197, p=.004\right)$ con el consumo de riesgo $\left(r_{s}=-.206, p=.003\right)$ y perjudicial en los jóvenes universitarios $\left(r_{s}=-.275, p=.001\right)$. Se concluye que a mayor sentido de coherencia, menor consumo de alcohol de riesgo y perjudicial.

\section{ABSTRACT}

The sense of coherence is one of the protective factors that is becoming important in the prevention of drug use. This is why to identify the relationship between the sense of coherence and the consumption of alcohol in university students is relevant. The design was descriptive correlational with stratified probabilistic sampling, the final sample was 227 young people enrolled in a private university. This study found that women have a high low-risk consumption and a risk consumption $30.3 \%$ and $33.3 \%$, respectively) compared to men who reported higher harmful consumption (59.1\%). A negative and significant correlation was found between the sense of coherence and the alcohol consumption measured by the AUDIT $\left(r_{s}=-.197, p=.004\right)$, with the risk consumption $\left(r_{s}=-.206 ; p=.003\right)$ and harmful consumption in university students $\left(r_{s}=-.275 ; p=.001\right)$. Based on the above, it is concluded at higher sense of coherence, lower risk and harmful alcohol consumption.

\section{INTRODUCCIÓN}

El consumo excesivo de alcohol en la actualidad es un grave problema de salud pública en virtud que en 2014 se le atribuyeron 3.3 millones de defunciones en el mundo según la Organización Mundial de la Salud o World Health Organization, en inglés (WHO, 


\section{InVESTIGACIÓn Y CIERCIA DE LA UחIVERSIDAD AUTÓnOMA DE RGUASCALIEחTES}

2014). Según el reporte mundial sobre el consumo de alcohol y salud (WHO, 2014), $5.1 \%$ de la carga mundial de enfermedades y lesiones, evaluada en Años de Vida Ajustados por Discapacidad (AVAD), se relacionan con el consumo excesivo de alcohol. Este indicador tiene mayor frecuencia en personas jóvenes, en quienes se reporta un incremento de consultas médicas y de hospitalizaciones como su consecuencia. Se indica que la ingesta de alcohol es el causante de más de 200 enfermedades, se asocia con el riesgo de desarrollar trastornos psiquiátricos y relacionados al comportamiento, donde se incluye la dependencia al consumo de alcohol, enfermedades no transmisibles (enfermedades hepáticas), diferentes tipos de neoplasias, enfermedades cardiacas, aunado a lo anterior se pueden presentar traumatismos asociados a la violencia y accidentes de tránsito (WHO, 2014).

El Informe de situación regional sobre el alcohol y la salud en las Américas de la Organización Panamericana de la Salud (OPS, 2015) reportó que en 2012 se presentó el fallecimiento de una persona cada 100 segundos en América, lo cual contribuyó al registró de más de 300,000 defunciones ese año; se indica que de no haber estado presente la ingesta de alcohol, aproximadamente 80,000 de estas muertes no se hubiesen presentado. En México, según los reportes de la Encuesta Nacional del Consumo de Drogas, Alcohol y Tabaco 2016-2017 (Instituto Nacional de Psiquiatría Ramón de la Fuente Muñiz, 2017), la población de 18 a 65 años arrojó que el consumo de alcohol alguna vez en la vida se mantuvo estable debido a que en 2011 fue de $71.3 \%$ y en 2016 fue de $71.0 \%$.

Con respecto a la prevalencia en el último año, tuvo una disminución significativa de dos puntos en 2011: de $51.4 \%$ a $49.1 \%$ en 2016 . No obstante, en la prevalencia en el último mes aumentó significativamente, de $31.6 \%$ en 2011 , a $35.9 \%$ en 2016 ; en relación con la prevalencia de consumo excesivo en el último mes creció significativamente de 2011, con $12.3 \%$, a 2016 , con $19.8 \%$. Al diferenciar por género se observa que la prevalencia mensual de consumo excesivo en hombres aumentó de $20.9 \%$ en 2011 a $29.9 \%$ en 2016 , esta tendencia se observó en mujeres de $4.1 \%$ en 2011 , a $10.3 \%$ en 2016 (Instituto Nacional de Psiquiatría Ramón de la Fuente Muñiz, 2017).

La etapa de adulto joven ( 18 a 25 años) se considera de mayor riesgo para la ingesta excesiva de alcohol. En esta etapa los jóvenes cursan los estudios universitarios; aumentan las responsabilidades, las demandas escolares, las exigencias de la sociedad y la familia. Es probable que los jóvenes en este periodo de su vida busquen tener estabilidad económica porque es frecuente se formalice la relación de pareja y el inicio de su propia familia, estos eventos pueden influenciar la ingesta excesiva de alcohol (Arnett, 2003; Díaz Heredia, 2012). Lo anterior afectaría en el corto plazo al ocasionar cambios negativos en sus conductas saludables de vida, dificultades en el rendimiento académico y aumento de la probabilidad de dependencia al alcohol.

La investigación con respecto al consumo de alcohol se ha centrado en los denominados factores de riesgo que aumentan la posibilidad de la ingesta (Becoña Iglesias, 2002). No obstante, existen factores de protección que pueden actuar como un escudo que retarde la iniciación y/o mantenimiento de esta conducta. El Sentido de Coherencia (SOC) es uno de los factores de protección que está cobrando importancia en la prevención del consumo de drogas (García-Moya, Jiménez-Iglesias, \& Moreno, 2013; Grevenstein, Bluemke, \& KroeningerJungaberle, 2016; Mattila et al., 2011; Menezes Coutinho \& Vilela Heimer, 2014; Rivera, García-Moya, Moreno, \& Ramos, 2013).

En 1987 Antonovsky planteó el Modelo Salutogénico que se concentra en recursos que permiten generar o mejorar la salud, como el SOC, el cual es un constructo definido a partir de lo que Antonovsky (1987) Ilamó Recursos Generales de Resistencia (GRRs); los mismos se definen como factores biológicos y psicosociales que permiten que las personas ejecuten un afrontamiento exitoso ante circunstancias y hechos de la vida que pueden generar estrés y ayuda a las personas a apreciar su vida como coherente, estructurada y comprensible (Antonovsky, 1987).

Este constructo se define como una orientación general que indica la medida en que una persona tiene un sentimiento de confianza interiorizado, permanente y a su vez dinámico, esta confianza aumenta la probabilidad de que los acontecimientos críticos o estresantes se resuelvan. Las personas con alto puntaje de SOC hacen uso de sus recursos generales de resistencia, los movilizan y emplean para resolver dificultades y para superar el estado emocional (Antonovsky, 1987; Lindström \& Eriksson, 2011).

El SOC se conforma de tres dimensiones: la comprensibilidad o capacidad de comprensión, 
se refiere a cómo aprende el individuo de los estímulos emanados de una fuente interna o externa de su existencia, la manejabilidad o de gestión se refiere a la percepción que el individuo desarrolla sobre sus recursos personales o sociales para cumplir exigencias trazadas por estos estímulos, y la capacidad de inversión o significatividad, que es la capacidad que el individuo tiene de dar sentido a los acontecimientos de la vida y encontrar en ello la razón para invertir energía y compromiso (Antonovsky, 1987). Se ha documentado en algunas poblaciones de jóvenes universitarios de África, Argentina, Colombia, Estados Unidos, Israel, Alemania, España, Finlandia y China que un nivel de SOC alto se relaciona negativamente con el consumo de alcohol, con el consumo excesivo y con los problemas asociados al uso de alcohol. Además, estos jóvenes presentan mayor habilidad para evitar o prevenir situaciones riesgosas, de esta manera reportan mayor probabilidad de incorporar a su vida conductas promotoras de salud (Antonovsky, 1987; García-Moya et al., 2013; Grevenstein et al., 2016; Malagón et al., 2012; Mattila et al., 2011; Menezes \& Vilela, 2014; Rivera et al., 2013).

Se ha descrito que las personas con un bajo puntaje de SOC tienen limitados recursos cognitivos para el afrontamiento efectivo ante situaciones difíciles de la vida y es probable que se involucren en conductas no saludables como el consumo excesivo de alcohol (Antonovsky, 1987). Es importante mencionar que hasta el momento no se ha localizado información científica sobre SOC y consumo de alcohol en jóvenes universitarios mexicanos, por lo que los autores consideran existe la necesidad de contar con conocimiento de la relación de estas variables, que tienen potencial para la promoción de la salud y protección de conductas de riesgo como el consumo de alcohol, por lo que los objetivos del estudio son: 1) identificar el tipo de consumo de alcohol por sexo y 2) determinar la relación del SOC y el consumo de alcohol en jóvenes universitarios.

\section{MATERIALES Y MÉTODOS}

El diseño del estudio fue descriptivo correlacional, la población de estudio se conformó por 953 jóvenes universitarios de ambos sexos que estudian en una universidad del área metropolitana de Monterrey, Nuevo León. El muestreo fue probabilístico estratificado por turnos (matutino y nocturno) con asignación proporcional al tamaño de estrato y dentro de cada estrato se empleó un muestreo sistemático con inicio aleatorizado de 1 en 4. La muestra se estimó bajo una correlación con una potencia de $84 \%$, un nivel de significancia $p<.05$ y un coeficiente de determinación de 0.06 (un efecto entre pequeño y mediano), la muestra final fue de 227 participantes (93 del turno matutino y 134 del nocturno).

La investigación fue aceptada por la Comisión de Ética para la investigación de la Facultad de Enfermería de la Universidad Autónoma de Nuevo León con número de registro FAEN-M-1271. Este cumplió lo estipulado por la Ley General de Salud en Materia de Investigación (SSA, 1987), tuvo el consentimiento informado de los jóvenes universitarios por el cual se aseguró su participación voluntaria, se protegió el anonimato, la confidencialidad y se precisó que tenían el derecho de retirarse del estudio en el momento que quisieran. Se obtuvo también la autorización de los directivos de la institución educativa para efectuar el proceso de selección y recolección de los datos.

Antes de la selección y recolección de datos el investigador y tres auxiliares previamente capacitados se colocaron en la entrada principal de la institución educativa y con un contador digital seleccionó de uno a cuatro con inicio aleatorio, en el turno matutino se comenzó con el primer participante y en el nocturno con el tercer sujeto. A la persona seleccionada se le invitó a formar parte del estudio indicando que los datos eran anónimos y confidenciales. Una vez que aceptó participar se condujo al espacio autorizado para entregar los instrumentos y el consentimiento informado, el cual fue firmado como forma de aceptación. Asimismo, se le explicó de forma clara y sencilla el alcance y los objetivos del estudio, así como las instrucciones para el llenado de los instrumentos de recolección y el tiempo necesario para contestar los mismos.

Se aplicó una cédula de datos personales y de consumo de alcohol para identificar datos sociodemográficos y de prevalencia de consumo de alcohol. Para medir el SOC se utilizó el Cuestionario Breve de Orientación hacia la Vida (SOC-13) derivado del Modelo Salutogénico compuesto por 13 ítems (Antonovsky, 1987) con el propósito de evaluar la capacidad de visualizar el SOC. Este cuestionario se deriva del cuestionario original de 29 ítems del mismo autor, el cual refirió que la versión breve del cuestionario permitiría contestar en un menor tiempo y así reducir la falta de respuesta; de la misma manera, se integra por tres dimensiones: comprensibilidad, 


\section{InVESTIGACIÓn Y CIEnCIA DE LA UחIVERSIDAD AUTÓnOMH DE RGUASCALIEחTES}

manejabilidad y significatividad. Este cuestionario en versión breve se ha utilizado en 48 países y en 49 lenguajes diferentes alrededor de todo el mundo, en diferentes edades, poblaciones, culturas y disciplinas. En 2014 la escala se aplicó en jóvenes universitarios mexicanos, se documentó un Coeficiente Alpha de Cronbach de 0.79 (Velázquez Jurado et al., 2014).

Para el consumo de alcohol se aplicó el Cuestionario de Identificación de los Trastornos debidos al Consumo de Alcohol (AUDIT) desarrollado por Babor y Grant (1989), adaptado en México por De la Fuente y Kershenobich (1992). Este instrumento se encuentra integrado por 10 preguntas con opción múltiple que evalúan el tipo de consumo de alcohol (sensato o sin riesgo, dependiente o de riesgo, dañino o perjudicial). Los reactivos 1 a 3 establecen la cantidad y frecuencia de consumo, y el consumo de bajo riesgo, los reactivos 4 a 6 el de riesgo y los ítems 7 a 10 el perjudicial. Este instrumento presenta una puntuación mínima de 0 y máxima de 40 , donde a mayor puntaje, mayor consumo; para el análisis estadístico se calcularon índices a los dos instrumentos. Los autores del instrumento indicaron una sensibilidad de $80 \%$ y una especificidad de $89 \%$; en 220 jóvenes universitarios del estado de Nuevo León los invetigadores Armendáriz García, Villar Luis, María Magdalena Alonso Castillo, Bertha Alicia Alonso Castillo y Oliva Rodríguez (2012) documentaron un Coeficiente Alpha de Cronbach de 0.84.

Los resultados se procesaron en el programa estadístico Statistical Package for the Social Sciences (SPSS) versión 21.0 para Windows. Se utilizó la estadística descriptiva e inferencial. Aunado a lo anterior, se aplicó la prueba de Bondad de Ajuste de Kolmogorov-Smirnov con Corrección de Lilliefors para es-

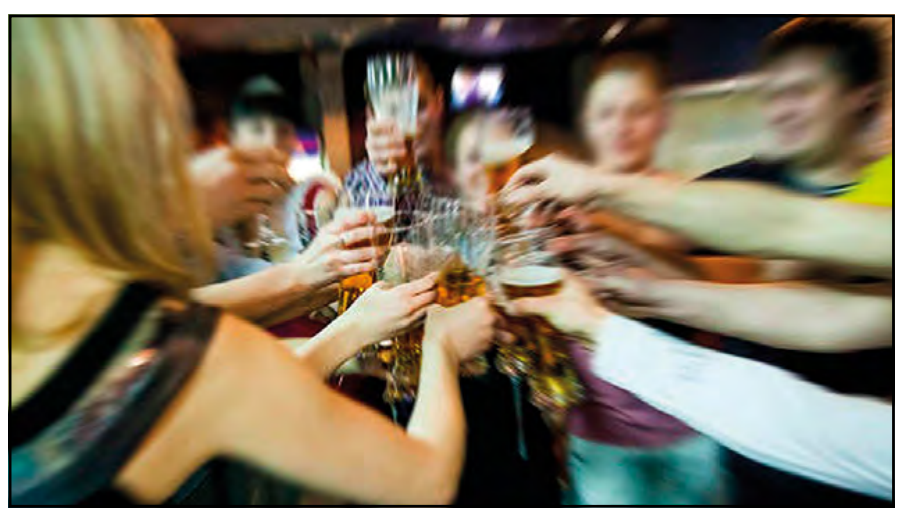

Figura 1. Consumo de alcohol en jóvenes universitarios. Imagen tomada de ELONCE (2017). tablecer la normalidad de las variables continuas y numéricas; la cual no presentó distribución normal, por lo que se utilizó estadística no paramétrica como el Coeficiente de Correlacion de Spearman para las relaciones bivariadas de índice de la escala SOC y del AUDIT y la prueba de Chi Cuadrada de Pearson $\left(\chi^{2}\right)$ para tipo de consumo de alcohol por sexo.

\section{RESULTADOS}

Enseguida se muestran los resultados referentes a las características sociodemográficas de los participantes del estudio, donde se identificó que $51.1 \%$ de estudiantes son del género masculino y $53.3 \%$ de los participantes tenía entre 18 y 20 años de edad, con una media de 20.7 años (DE= 1.8). Con respecto al grado escolar, $26.9 \%$ cursan los primeros semestres de la carrera universitaria con un promedio de 13.2 $(D E=1.0)$ años de escolaridad; asimismo, se observó que $94.7 \%$ son solteros y que $53.7 \%$ de los participantes estudia y trabaja. Con respecto al tipo de empleo de los participantes, $23.8 \%$ trabaja en institución pública, $59.0 \%$ en privada, $9.0 \%$ trabaja por su cuenta y $8.2 \%$ en servicios como meseros, cocineros y ayudantes; en cuanto a la cantidad de horas que dedicaban a trabajar, además de estudiar, reportaron una media de $23.3 \mathrm{~h}$ a la semana (DE= 18.1).

En la tabla 1 se obtuvieron las prevalencias de consumo global (alguna vez en la vida), prevalencia lápsica (en el último año), actual (último mes) e instantáneo (últimos siete días). Se destaca que todos los participantes reportaron haber tomado alcohol alguna vez en la vida. Se destacan altas proporciones de consumo, tanto para la prevalencia actual (78\%) como para la instantánea (48\%).

Tabla 1

Prevalencia de consumo de alcohol

\begin{tabular}{lcccccc}
\hline Variable & \multicolumn{3}{c}{ Sí } & \multicolumn{2}{c}{ No } & \multicolumn{2}{c}{ IC 95\% } \\
\cline { 2 - 7 }$n^{b}=209$ & $f$ & $\%$ & $f$ & $\%$ & LI & LS \\
Lápsica & 209 & 92 & 18 & 8 & 89 & 96 \\
Actual & 178 & 78 & 49 & 22 & 73 & 84 \\
Instantánea & 109 & 48 & 118 & 52 & 41 & 55 \\
\hline
\end{tabular}

Nota: $n^{b}=$ consumidores de alcohol en el último año, $f=$ frecuencia, $\%=$ porcentaje, IC 95\% = Intervalo de Confianza al 95\%, LI= Límite Inferior, LS= Límite Superior. Elaboración propia.

En relación con el primer objetivo del estudio, en la tabla 2 se observa que existen diferencias significativas para el tipo de consumo por sexo $\left(\chi^{2}=\right.$ 11.07, $p<.001$ ), se observa que el consumo de bajo 
Tabla 2

Chi Cuadrada de Pearson $\left(\chi^{2}\right)$ para tipo de consumo de alcohol por sexo

\begin{tabular}{|c|c|c|c|c|c|c|c|c|}
\hline $\begin{array}{l}\text { Variable } \\
n^{b}=209\end{array}$ & & & & $\begin{array}{l}\text { umo } \\
\text { esgo }\end{array}$ & & $\begin{array}{l}\text { nsumo } \\
\text { judicia }\end{array}$ & \multirow[b]{2}{*}{$\chi^{2}$} & \multirow[b]{2}{*}{$p$} \\
\hline & $f$ & $\%$ & $f$ & $\%$ & $f$ & $\%$ & & \\
\hline Femenino & 30 & 30.3 & 33 & 33.3 & 36 & 36.4 & 11.07 & .004 \\
\hline Masculino & 19 & 17.3 & 26 & 23.6 & 65 & 59.1 & & \\
\hline
\end{tabular}

Nota: $f=$ frecuencia, $\%=$ porcentaje, $\chi^{2}=$ Chi cuadrada de Pearson, $p=$ significancia $n^{\mathrm{b}}=$ consumidores de alcohol en el último año.

Elaboración propia.

Tabla 3

Coeficiente de Correlación de Spearman para el SOC y Consumo de Alcohol

\begin{tabular}{llc}
\hline Variable & \multicolumn{2}{c}{ Índice de SOC } \\
\cline { 2 - 3 }$n=227$ & $\mathrm{r}_{\mathrm{s}}$ & $\mathrm{p}$ \\
\hline Edad de inicio de consumo & -.048 & .473 \\
Índice AUDIT & $-.197^{* *}$ & .004 \\
Índice consumo de bajo riesgo & -.051 & .467 \\
Índice consumo de riesgo & $-.206^{* *}$ & .003 \\
Índice consumo perjudicial & $-.275^{* *}$ & .001 \\
\hline
\end{tabular}

Nota: $n=$ muestra total, $n^{b}=$ consumidores de alcohol en el último año, $p=$ significancia, ${ }^{* *} p<.01, r_{s}=$ Coeficiente de Correlación de Spearman. Elaboración propia.

riesgo (30.3\%) y el de riesgo es mayor en el sexo femenino (33.3\%); sin embargo, con respecto al consumo perjudicial, es superior en el sexo masculino (59.1\%).

En lo referente a la relación entre las variables del presente estudio los resultados se reportan en la tabla 3, se muestra que existe una correlación negativa y significativa de la escala de SOC con la escala AUDIT $\left(r_{s}=-.197, p=.004\right)$, con el consumo de alcohol de riesgo $\left(r_{s}=-.206, p=.003\right)$ y con el consumo perjudicial $\left(r_{s}=-.275, p=.001\right)$.

\section{DISCUSIÓN}

En los participantes del presente estudio se identificaron prevalencias elevadas de consumo de alcohol, estos datos son mayores a lo indicado por la Encuesta Nacional de Drogas, Alcohol y Tabaco, ENCODAT 2016-2017 (INPRFM, 2017), donde se mostró que la prevalencia en el último año es de $55.7 \%$ y el consumo en el último mes de 35.9\%. Esta situación quizá se explique por tener mayor acceso a la substancia debido al incremento de grupos con los cua- les socializa, así como el incremento de actividades sociales que frecuentemente se encuentran relacionadas con el consumo de alcohol, asimismo, en esta edad para los jóvenes es muy accesible la compra de estas sustancias (Cabrejas Martínez, Llorca Diez, Gallego Álvarez, Bueno Carrera, \& Diez Sánchez, 2014; García, Calvo, Carbonell, \& Giralt, 2017; Góngora Soberanes \& Leyva Piña, 2005). Aunado a lo anterior, pueden ocurrir situaciones dentro de sus actividades escolares que pueden generar estrés y ser afrontadas mediante el consumo de alcohol (Armendáriz García et al., 2012).

En relación con las diferencias entre los tipos de consumo de alcohol y el sexo, estos datos son similares a lo reportado por Mendes Moutinho, De Oliveira Cruz Mendes y Lopes (2015), quienes documentaron diferencias significativas donde los participantes masculinos reportaron mayor consumo perjudicial. Lo anterior puede explicarse probablemente como parte de las premisas socioculturales, donde es más aceptado por parte de los padres y la sociedad el consumo excesivo en varones y se espera un control del consumo femenino debido a las creencias arraigadas en la sociedad (Góngora Soberanes \& Leyva Piña, 2005).

Sin embargo, con respecto al consumo de riesgo, es preocupante que en las mujeres se está igualando con el de los varones, probablemente porque las jóvenes tengan una percepción de equidad de género en la que pretenden reproducir patrones de conducta de los hombres, como el consumo de alcohol, entre otras razones, como la búsqueda de placer o diversión; sin embargo, las diferencias biológicas de tipo metabólico y hormonal en las mujeres pueden afectar su salud en menor tiempo (BriñezHorta, 2001).

Los hallazgos de este estudio ratifican lo documentado en la literatura por varios investigadores (García-Moya et al., 2013; Grevenstein et al., 2016; Mattila et al., 201 1; Mendes Moutinho et al., 2015; Tobamidanik \& Zabkiewicz, 2009), donde reportan que un alto puntaje de SOC se asocia con un menor consumo de alcohol; lo anterior indica que actúa como factor de protección al limitar el consumo 


\section{IIVESTIGAGIÓn Y CUERCIA DE LA UNIVERSIDAD AUTÓnOMF DE RGUASCHLIETTES}

de alcohol de riesgo y perjudicial. Probablemente un alto SOC mejora la capacidad del joven universitario para comprender y encontrar un significado a las circunstancias o eventos de la vida que pueden ser estresantes, se adapta y afronta las situaciones difíciles y evita el peligro, por lo que existe una mayor probabilidad de que incorporen a su vida conductas promotoras de salud (Antonovsky, 1996). Además, el SOC permite a los individuos identificar los recursos que tienen disponibles para afrontar los acontecimientos día a día y distinguir aquellos que son merecedores de inversión y compromiso.

\section{Limitaciones del estudio}

Dentro de las restricciones del presente estudio se encuentra la aplicación de la versión breve del Cuestionario de Orientación hacia la vida (SOC-13), debido a que sería importante aplicar también el cuestionario de Orientación hacia la vida (SOC-14) en su extensión original para identificar si existe alguna variación con respecto a la medición de la variable. Otra limitación es relacionada con el diseño transversal del estudio, el cual no permite obtener información más amplia relacionada con el fenómeno de estudio, por lo cual se recomienda realizar estudios longitudinales en futuras investigaciones.

\section{CONCLUSIONES}

Se identificaron diferencias significativas en el consumo por género. Se documentó que el consumo de bajo riesgo y el riesgoso (33.3\%) es mayor en el género femenino, mientras el perjudicial $(59.1 \%)$ es mayor en el masculino. Asimismo, se identificó una correlación negativa y significativa entre el SOC y el AUDIT, así como entre el consumo de riesgo y el perjudicial.

Se concluye que es necesario profundizar en el estudio de SOC y consumo de alcohol en adultos jóvenes que no estudien para conocer si los hallazgos de este estudio son consistentes. El diseño de intervenciones multidisciplinarias preventivas y protectoras de consumo de alcohol es una necesidad prioritaria de los sistemas de salud, en virtud del incremento y consecuencias de esta conducta en adultos jóvenes. En un futuro se podrían desarrollar intervenciones preventivas fortaleciendo el SOC que incrementen la comprensión, explicación y otorgamiento de significado a eventos de la vida y problemas, al encontrar los recursos generales de resistencia con los que cuenta el individuo para tomar decisiones enfocadas a comportamientos saludables.

\section{REFERENCIAS}

- Antonovsky, A. (1987). Unravelling the mystery of health: How people manage stress and stay well. San Francisco: JosseyBass.

(1996). The salutogenic model as a theory to guide health promotion. Health Promotion International, $11(1), 11$ 18.

- Armendáriz García, N. A., Villar Luis, M. A., Alonso Castillo, M. M., Alonso Castillo, B. A., \& Oliva Rodríguez, N. N. (2012). Eventos estresantes y su relación con el consumo de alcohol en estudiantes universitarios. Investigación en Enfermería: Imagen y Desarrollo, 14(2), 97-112.

- Arnett, J. J. (2003). Conceptions of the transition to adulthood among emerging adults in American ethnic groups: Exploring cultural conceptions of the transitions to adulthood. New Directions for Child and Adolescent Development, 2003(100), 63-76.

- Babor, T. F., \& Grant, M. (1989). From clinical research to secondary prevention: International collaboration in the deve- lopment of the Alcohol Disorders Identification Test (AUDIT). Alcohol Health and Research World, Fall 1989, 371-374.

- Becoña Iglesias, E. (2002). Bases científicas de la prevención de las drogodependencias. Madrid: Delegación del Gobierno para el Pan Nacional contra las drogas.

- Bríñez-Horta, J. A. (2001). Diferencias de género en problemas con el alcohol, según el nivel de consumo. Adicciones, 13(4), 439-455.

- Cabrejas Martínez, B., Llorca Diez, A., Gallego Álvarez T., Bueno Carrera, G., \& Diez Sánchez, M. A. (2014). Hábitos de consumo de alcohol en población universitaria. Historia y Comunicación Social, 19(esp. marzo), 777-789.

- De la Fuente, J. R., \& Kershenobich, D. (1992). El alcoholismo como problema médico. Revista Facultad de Medicina UNAM, 35(2), 47-51.

- Díaz Heredia, L. P. (2012). Promoción de salud: Autotrascendencia, espiritualidad y bienestar en no consumidores y consumidores moderados de alcohol (Tesis doctoral). Recuperada de http://bdigital.unal.edu.co/8050/ 
- ELONCE. (2017). Adolescentes: La mitad bebe alcohol en exceso y creció el consumo de marihuana [Fotografía]. Recuperada de https://www.elonce.com/secciones/ sociedad/511664-adolescentes-la-mitad-bebe-alcohol-enexceso-y-crecin-el-consumode-marihuana.htm

- García, C., Calvo, F., Carbonell, X., \& Giralt, C. (2017). Consumo intensivo de alcohol y conductas sexuales de riesgo en población universitaria. Health and Addictions, 17(1), 63-71.

- García-Moya, I., Jiménez-Iglesias, A., \& Moreno, C. (2013). Sense of coherence and substance use in Spanish adolescents. Does the effect of SOC depend on patterns of substance use in their peer group? Adicciones, 25(2), 109-117.

- Góngora Soberanes, J., \& Leyva Piña, M. A. (2005). El alcoholismo desde la perspectiva de género. El Cotidiano, 132, 84-91.

- Grevenstein, D., Bluemke, M., \& Kroeninger-Jungaberle, H. (2016). Incremental validity of sense of coherence, neuroticism, extraversion, and general self-efficacy: Longitudinal prediction of substance use frequency and mental health. Health and Quality of Life Outcomes, 14(9). doi: 10.1186/s12955-0160412-z

- Instituto Nacional de Psiquiatría Ramón de la Fuente Muñiz. (2017). Encuesta Nacional de Consumo de Drogas, Alcohol y Tabaco, ENCODAT 2016-2017. Reporte de Alcohol [Documento en pdf]. Recuperado de https://drive.google.com/ file/d/1rMIKaWy34GR51sEnBK2-u2q_BDK9LA0e/view

- Lindström, B., \& Eriksson, M. (2011). Guía del autoestopista salutogénico. Camino salutogénico hacia la promoción de la salud. Girona: Documenta Universitaria.

- Malagón-Aguilera, M. C., Fuentes-Pumarola, C., Suñer-Soler, R., Bonmatí-Tomàs, A., Fernández-Peña, R., \& Bosch-Farré, C. (2012). El sentido de coherencia en el colectivo enfermero. Enfermería Clínica, 22(4), 214-218.

- Mattila, M. L., Rautava, P., Honkinen, P. L., Ojanlatva, A., Jaakkola, S., Aromaa, M.,... Sillanpää, M. (2011). Sense of cohe- rence and health behaviour in adolescence. Acta Paediatrica, 100(12), 1590-1595.

- Mendes Moutinho, L. S., De Oliveira Cruz Mendes, A. M., \& Lopes, M. J. (2015). Alcohol consumption and the sense of coherence in young people in educational training. SMAD. Revista Eletrônica Saúde Mental Álcool e Drogas, 11 (4), 208-216.

- Menezes Coutinho, V. M., \& Vilela Heimer, M. V. (2014). Sense of coherence and adolescence: An integrative review of the literature. Ciência \& Saúde Coletiva, 19(3), 819-827.

- Organización Panamericana de la Salud (2015). Informe sobre la situación regional sobre el alcohol y la salud en las Américas [Informe en pdf]. Washington, DC: Autor. Recuperado de https://www.paho.org/hq/dmdocuments/2015/alcohol-Informe-salud-americas-2015.pdf

- Rivera, F., García-Moya, I., Moreno, C., \& Ramos, P. (2013). Developmental contexts and sense of coherence in adolescence: A systematic review. Journal of Health Psychology, 18(6), 800-812.

- Secretaría de Salud. (1987). Reglamento de la Ley General de Salud en Materia de Investigación para la salud. En Ley Ge-

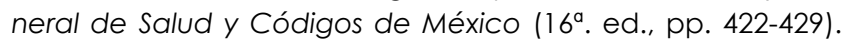
México: Porrúa.

- Tobamidanik, L., \& Zabkiewicz, D. (2009). Indicators of sense of coherence and alcohol consumption-related problems: The 2000 US national alcohol survey. Substance Use \& Misuse, $44(3), 357-373$.

- Velázquez Jurado, H., Cárdenas Rivera, V., Chávez Franco, A., Oliva Montes de Oca, V., Hernández Salazar, P., \& Pulido Rull, M. A. (2014). Comparación de dos formas de una escala de sentido de coherencia. Revista Intercontinental de Psicología y Educación, 16(2), 51-70.

- World Health Organization. (2014). Global status report on alcohol and health-2014 ed. [Reporte en pdf]. Geneva, Switzerland: WHO. Recuperado de http://www.who.int/substance abuse/publications/global_alcohol_report/msb_gsr_2014_1. pdf? $\cup a=1$ 